\title{
La variedad de los ideales orientales: una relectura de la unidad de Asia en la obra de Okakura Kakuzō desde la perspectiva india
}

\author{
Sergio Román Aliste \\ Universidad Rey Juan Carlos \\ sergio.aliste@urjc.es
}

RESUMEN: Este estudio propone nuevos matices en la interpretación de la obra de Okakura Kakuzō The ldeals of the East (1903) a partir de fuentes teóricas bengalíes ligadas a la enseñanza artística. La interacción de Okakura Kakuzō con las élites culturales de Calcuta propició no solo la redacción de ese libro, sino también la puesta en marcha de un hermanamiento cultural de gran interés para el estudio de la modernidad artística en Asia. Este artículo descompone esa unidad ideológica de Asia propuesta por Okakura en interacción con los teóricos bengalíes con la intención de exponer las principales fuentes teóricas occidentales utilizadas en la construcción del ideal unitario asiático. La finalidad de este artículo es aportar claves ya consolidadas en la teoría artística anticolonial india para la lectura del texto de Okakura en una clave más abierta.

PALABRAS CLAVE: Okakura Kakuzō; Rabindranath Tagore; Teoría artística; Panasianismo; Originalidad; Tradición; Escuela de Bengala.

\section{The Diversity of Oriental Ideals: a Re-reading of Asian Unity in the Work of Okakura Kakuzō from the Indian Perspective}

ABSTRACT: This paper proposes new nuances in the interpretation of Okakura Kakuzō's work The ldeals of the East (1903) using Bengali theoretical sources linked to artistic pedagogy. The interaction of Okakura Kakuzō with the cultural elites of Calcutta led to the writing of that book, but also to the implementation of a cultural twinning relevant to the study of artistic modernity in Asia. This article breaks down the ideological unity of Asia proposed by Okakura and the Bengali theorists with the intention of showing the main Western theoretical sources used in the construction of the Asian unitary ideal. The purpose of this article is to provide already consolidated clues in Indian anticolonial artistic theory to interpret Okakura's text in a more open key.

KEYWORDS: Okakura Kakuzō; Rabindranath Tagore; Artistic theory; Pan-Asianism; Originality; Tradition; Bengal School.

Recibido: 28 de febrero de 2020 / Aceptado: 25 de junio de 2020.

Calcuta, encrucijada de relecturas occidentalizadas del arte asiático

La ciudad de Calcuta fue el centro protagonista de una serie de encuentros e interacciones cruciales para la cultura y las artes de principios del siglo XX. Un papel, el de la gran urbe bengalí, no solo fundamental para el contexto colonial indio o el relativo a la modernidad asiática, sino también crucial para la comprensión de la realidad artística de Asia desde la perspectiva del Atlántico Norte. Los procesos particulares, algunos de los cuales son anotados en el presente artículo, han despertado gran interés académico en las últimas dos décadas, aunque la repercusión global de Calcuta como uno de los epicentros cultura-

Cómo citar este artículo: ROMÁN ALISTE, Sergio, «La variedad de los ideales orientales: una relectura de la unidad de Asia en la obra de Okakura Kakuzō desde la perspectiva india", Boletín de Arte-UMA, n. ${ }^{\circ}$ 42, Departamento de Historia del Arte, Universidad de Málaga, 2021, pp. 247-255, ISSN: 0211-8483, e-ISSN: 2695-415X, DOI: http://dx.doi.org/10.24310/BoLArte.2021.vi42.8145 
les de las primeras décadas del XX aún está pendiente de ser ponderada. Los personajes protagonistas de esta serie de confluencias, epígonos románticos y eruditos en sus respectivos campos, actuaron movidos por los valores utópicos del renacimiento cultural de las tradiciones orientales entendidas como un todo coherente: podría hablarse de un tiempo de ideales y un anhelo profundo de unidad. El eje de esta serie de encuentros fue la familia Țhākur, cuyo apellido fue adaptado a la fonética inglesa como Tagore, que ejerció como anfitriona para el estudioso japonés Okakura Kakuzō ${ }^{-1}$ entre los años 1901-1902 y 1912, al igual que lo hizo para el historiador del arte Ananda Kentish Coomaraswamy (1909-1913) durante sus respectivas estancias en Calcuta ${ }^{2}$. El más famoso de los miembros del clan Tagore fue Rabindranath, posteriormente galardonado con el Premio Nobel de Literatura (1913). Este autor, alineado ideológicamente con los propósitos generales de Okakura y Coomaraswamy en la consideración cultural de Asia, ejerció de gran embajador ante Occidente durante el periodo de entreguerras, perpetuando y complementando un ideario perfectamente articulado que se desarrolla a continuación. Los tres escritores teorizaron acerca del Continente asiático en términos unitarios, tanto en lo artístico como en lo estético, y los dos primeros apuntaron adicionalmente a un sentido más político e intangible: el de la superioridad espiritual y moral de Asia con respecto a Occidente.

Hay una frase ubicua en cualquier estudio sobre Okakura Kakuzō, especialmente en aquellos que abordan la interacción del autor japonés con India. Se trata de la sentencia «Asia is one» (Okakura, 1905: 1), máxima inaugural de su obra The Ideals of the East (London, John Murray, 1903), y al mismo tiempo proclama fundacional del panasianismo como movimiento cultural anticolonial ${ }^{3}$. Como ha destacado John Clark, las ideas rectoras de este texto son, entre otras: a) la consideración de Asia como una continuidad en sus diferencias, en parte solo comprensible o asimilable por sus propios habitantes; b) la defensa de Japón como territorio depositario del legado unitario de Asia a lo largo de los siglos y c) la creencia de que ese pasado, custodio de la esencia cultural, debía ser revisado para la regeneración cultural del continente (Clark, 2005: 10-11). Japón retiene todo el protagonismo y el peso de la acción en el planteamiento de Okakura Kakuzō, pero en gran medida catalizado por el impacto de India y el descubrimiento experiencial de sus afinidades comunes con la cultura del Asia meridional, en concreto a través del vínculo budista. La interconexión del continente asiático, particularmente de los ámbitos indo-sino-nipón, fue defendida por Okakura hasta el final de su vida (Nagahiro, 2012: 35), y su legado es rastreable en posteriores hermanamientos como el de los cineastas Satyajit Ray y Akira Kurosawa décadas más tarde 4 .

Como afirma Yayoi Kawamura en el prólogo a la reciente traducción de The Ideals of the East al castellano (Okakura, 2018: 20-21), el texto en cuestión fue dedicado al pueblo indio, fruto del apasionado encuentro con su cultura, y escrito directamente en inglés para ser publicado en Londres, dirigido por tanto al lector occidental. Debe hablarse, en este sentido, de un manifiesto ligado a la agitación cultural y al afianzamiento romántico del hermanamiento indo-japonés. Pero tal encuentro, o pretendido reencuentro, cargado de intencionalidad antiacadémica y rebeldía frente a las historiografías británicas del arte indio, era no obstante deudor de los prismas académicos decimonónicos, tomados a conveniencia para la acción combativa.

Los respectivos contextos indio y japonés compartían estrategias y propósitos en la revitalización de los valores artísticos tradicionales, e incluso se ha sugerido una correspondencia de roles entre ambos contextos: Abanindranath Tagore sería en el espacio bengalí lo que el pintor Yokoyama Taikan fue para Japón; el académico Ernest Fenollosa actuaría en el ámbito nipón del mismo modo que el historiador y gestor británico Ernest Binfield Havell para el arte indio; el llamado Club Vicitrā de Jorasanko (Calcuta) sería un obvio paralelo de la escuela Nihon Bijutsuin; y finalmente Rabindranath Tagore tuvo un papel con respecto al arte indio equivalente al que Okakura Kakuzō tuvo para el japonés (Guha-Thakurta, 2009: 24-26). Por otra parte, la razón de la presencia de Okakura Kakuzō en Calcuta debía mucho a la capacidad inspiradora de Swami Vivekananda, cuya discípula, Margaret Noble, escribió el prólogo a The Ideals of the East (Román, 2013: 787-788). Entre las palabras introductorias de Margaret Noble, conocida como Sister Nivedita, había otra comparación de roles, puesto que para esta autora Okakura Kakuzō sería para Japón el William Morris de Arts and Crafts, y del mismo modo Nihon Bijutsuin sería un equivalente oriental de Merton Abbey (Okakura, 1905: viii-ix). Las correspondencias apuntadas no solo explican un hermanamiento o sintonía, sino también evidencian la bicefalia cultural que las élites occidentalizadas estaban acostumbradas a ejercer, como 
mediadores entre dos ámbitos no conciliables sin contradicciones. El historiador Partha Chatterjee se ha aproximado a esta cuestión afirmando que las élites nacionalistas en contextos coloniales se debaten en una bipolaridad entre la occidentalización y el tradicionalismo, actuando desde un ámbito y hablando o expresándose desde el opuesto (2011: 4). Por ejemplo, Dipesh Chakrabarty recoge algunas afirmaciones de autores bengalíes coetáneos a Rabindranath Tagore, en las que se afirma que «las obras de Rabindranath son literatura europea escrita en lenguaje bengalí», o «En sus manos [de Rabindranath] la literatura bengalí se tornó occidental en todo excepto en la lengua» (Chakrabarty, 2008: 215). El mismo escenario se muestra en el caso de Okakura Kakuzō, con una famosa frase que dirigió a su hijo: «te sugiero que vistas kimono cuando viajes al extranjero si piensas que tu inglés es suficientemente bueno; pero nunca vistas atuendo japonés si hablas un inglés imperfecto» (Guth, 2000: 623).

El enmascaramiento que se deduce de este tipo de actitud, y que es equivalente tanto en los principales protagonistas japoneses como en los bengalíes, puede ser desentrañado desde las claves que proporciona el propio hermanamiento panasiático. La retórica de profundización en la esencia tradicional asiática tiene una base de aproximación fuertemente occidentalizante, en gran medida para lograr la seducción y comprensión del interlocutor atlántico, como es el caso de The Ideals of the East. Como ya se ha apuntado en diversos estudios, el enfoque de Okakura debe su dualismo occidental-oriental a una base hegeliana, que oponía materialismo europeo al espiritualismo asiático (Notehelfer, 1990: 331). El planteamiento del presente estudio defiende que, para la interpretación de los discursos artísticos bengalíes en torno a la familia Tagore es especialmente esclarecedora la incorporación de las fuentes japonesas del ámbito de Okakura Kakuzō, y viceversa. En las siguientes líneas se aportan algunas claves obtenidas desde la teoría artística del nacionalismo cultural bengalí, pretendiendo iluminar con ello la posición de Okakura Kakuzō en The Ideals of the East.

Un satélite japonés para Calcuta: la teoría artística de Śāntiniketan y el magisterio de Okakura Kakuzō

Uno de los lugares que Okakura Kakuzō visitó en su estancia bengalí de 1901-02 fue Śāntiniketan (Hay, 1970: 38), una finca rural perteneciente a la familia Tagore en la que justo por aquellas fechas Rabindranath había fundado una humilde escuela experimental. El experimento educativo de Rabindranath Tagore fue ganando peso y ambición académica hasta lograr constituirse en universidad de las artes unos dieciocho años más tarde. Allí, en 1919 fundó Kalābhavan, una facultad de artes plásticas que pasó a estar dirigida por Nandalal Bose, un artista protagonista de la interacción del círculo japonés de Okakura con la familia Tagore en la segunda estancia de 1912.

En el año 1912 Nandalal Bose era aún un alumno aventajado del círculo de Abanindranath Tagore, cuando Okakura recaló de nuevo en Calcuta después de su periodo de residencia en Boston. Okakura aportó algunos consejos formativos al joven Nandalal que no aparecen recogidos en fuentes escritas del autor japonés, pero cuya fortuna fue evidente en el contexto bengalí. Esas pautas fueron transmitidas oralmente como una fórmula magistral en Śāntiniketan hasta que fueron articuladas más tarde como la base de una teoría artística de tono panasiático.

La mencionada receta no era sino un compendio de tres ingredientes esenciales para la creación artística, según el criterio de Okakura. El ideal de Okakura Kakuzō, tal como fue asimilado por Nandalal Bose comprendería «estudiar la naturaleza, estudiar el pasado y conocerse y ser uno mismo», y como tal ha sido elevado a la categoría de ideal supremo en la construcción teórico-artística de Sāntiniketan (Department of History of Art, 1979: 2). Esos tres elementos conformarían un triángulo aparentemente totalizador, y esas tres aristas serían naturaleza, tradición y originalidad; tres vértices a los que se les exigiría -previsiblemente- un equilibrio tácito. Podría tomarse como referencia la propia opinión de Nandalal Bose al respecto, tal como fue recogida por su amigo Kanai Samanta:

Okakura solía decir que la perfección en el arte consiste en estos tres elementos: Naturaleza, Tradición y Originalidad. Cuando carece del estudio del natural, el arte se vuelve endeble y artificial; sin el conocimiento de la tradición, el arte se muestra como algo embrionario y propio de aficionados; sin originalidad, además de rígido y exánime, el arte carecería de su raison d'être. Por otro lado, si se basa solo en la naturaleza, el arte se vuelve mera imitación; si se dedica en exclusiva al seguimiento de la tradición, el arte no se elevará por enci- 
ma de la artesanía; y finalmente si no se cuenta más que con la originalidad como guía, el artista se comportaría casi como un loco (Samanta, 1982: 5-6; trad. propia).

El equilibrio que esta afirmación propone, considerando la creación artística como una vía intermedia entre el exceso y el defecto de esos tres componentes, no es tampoco ajeno a una ponderación adecuada de esa tríada. En términos formativos, los niveles más avanzados de la enseñanza en Kalābhavan se articulaban en torno a tres tipos de trabajos: el «trabajo original», el «estudio del natural» y la «copia tradicional». Los alumnos eran animados a saltar de un modo a otro, a fin de mantener su entusiasmo creativo (Guha-Thakurta, 2008: 310-311). No obstante, aunque la concordancia entre ese tipo de trabajos y los tres ejes que se acaban de enunciar pueda parecer evidente, el calado de la tríada de Okakura se abordará en base a una perspectiva más ideológica, desde el punto de vista de la cimentación teórica. Sin embargo, desde un enfoque revisionista, al menos desde la posición que aporta Śāntiniketan, no puede considerarse que se trate de un triángulo equilátero, en el que cada una de las aristas conlleve un número equiparable de grados. La forma de este polígono correspondería más bien, desde la interpretación aquí propuesta, con la de un triángulo escaleno dominado por la amplitud obtusa de uno de sus vértices y la diferenciada agudeza de las dos aristas restantes.

La lectura historiográfica previsible podría dar una mayor importancia a la tradición, y más aún en un contexto de construcción identitaria nacionalista como el caso bengalí, en el que el peso de los valores del pasado y la eventual pervivencia incorrupta en el presente darían pie a otorgar a ese pilar un protagonismo incuestionable. La cuestión de la tradición tiene, sin lugar a dudas y como se pondrá de relieve en las siguientes páginas, un peso crucial en tal apreciación, pero la postura aquí defendida otorga al tercero de los ángulos, el de la originalidad (especialmente a los componentes que se esconden tras ese término) el papel fundamental, pues articula no ya solo los otros dos elementos de ese triángulo, sino cualquier otro elemento implicado en la teoría artística del contexto bengalí, y en especial en la de Śāntiniketan. Como se verá, no se tratará tanto de creación en términos de novedad, sino más bien de lectura esencialista: de acceso al origen desde un rol necesariamente imaginativo.
Śāntiniketan como receptáculo ideal del triángulo «naturaleza-tradición-originalidad»: los ideales con los que Oriente debe renacer

El primero de los componentes del ideal creativo de Okakura remitía a la naturaleza, o al menos así fue como Nandalal lo difundió en la teoría y la práctica artística en torno a Kalābhavan. Kapila Vatsyayan alude al ideal de Okakura como una oportunidad para la aplicación rigurosa de una terminología historiográfica que pueda superar fronteras dualistas, como se anotó anteriormente. Con respecto al primero de los componentes, la autora aludía a la concepción fuertemente interdependiente en el arte indio entre hombre y naturaleza, así como entre arte y naturaleza (Vatsyayan, 1984: 146-148). Aunque la historiografía de Śāntiniketan ha consagrado a la «naturaleza» como uno de los ejes de ese triángulo, habría motivos para pensar que Okakura no le transmitió a Nandalal ese término puntual como abstracción del mundo sensible, sino como proceso creativo de observación. Abanindranath Tagore, guru de Nandalal en el momento de la segunda visita de Okakura (hacia 1912), relató al final de su vida los recuerdos de la visita del crítico japonés a la mansión de Jorasanko, dentro de su ensayo autobiográfico Jorāsāñkor Dhāre (1944). Y en medio de la evocación de aquella segunda estancia de Okakura, el pintor bengalí citó los tres principios tal como el crítico japonés debió expresarlos, en inglés, y los transcribió adaptándolos fonéticamente a la grafía bengalí sin tratar de volcarlos en algún vocablo nativo equivalente: los tres principios serían observación, tradición y originalidad ${ }^{5}$. La naturaleza sería, por tanto, no una entidad sino una pauta de aproximación desde la contemplación activa del artista. Con respecto al segundo de los elementos, la tradición, toda lectura en el contexto bengalí, incluso con la finalidad de esclarecer los textos de Okakura Kakuzō, debe tener en cuenta los textos de Ananda Coomaraswamy. La recuperación textual y terminológica fue clave en la configuración de la imagen de la tradición artística vernácula bengalí. Sin embargo, la postura de Coomaraswamy no nace de una posición terminológica de rigor aséptico, sino que se apoya en el uso erudito y personalista de los términos, forzando en numerosas ocasiones traducciones interesadas, como la neoplatónica versión de rasa como beauty ${ }^{6}$. Por tanto, esa visión indiferenciada de los cauces de la tradición responde más bien a ese intento 
ya anotado de construcción de una tradición ideal, en la que no existiría diferenciación en las artes, pero no a causa de que la terminología sánscrita favorezca tal postura de forma unívoca, sino porque esa era una posición opuesta a la división de las artes europea (Fernández Gómez, 2008) y en ese sentido era especialmente útil como herramienta ideológica anticolonial, aunque Coomaraswamy participara inevitablemente de aquella división.

Restaría solo un componente, el aparentemente menos «oriental» de los tres, y el que responde obviamente a la compatibilidad que Okakura Kakuzō encontraba entre el seguimiento del pasado y la proyección individualista hacia el progreso artístico (Mitter, 1994: 265). La posición de Coomaraswamy, por su parte, contemplaba con extrema dificultad el individualismo y la expresión sentimental del artista moderno ${ }^{7}$; era por tanto fuertemente reticente hacia el tercer vértice del triángulo, el de la originalidad, al menos desde el enfoque de lo «novedoso» ${ }^{8}$. Pero ello mismo le conduce a una posición híbrida en los textos contenidos en Essays on National Idealism (1909), pues necesitaba recurrir a la exégesis postromántica para huir del ideal de la originalidad como novedad, y al mismo tiempo, conseguir encontrar en ella las claves explicativas de una cuestión candente: cómo debe funcionar la creatividad del artista asiático moderno para que sus obras gocen de la misma vitalidad que el arte del pasado ${ }^{9}$. En uno de esos ensayos -justo en la época de mayor cercanía a Nandalal Bose- Coomaraswamy recurría a John Ruskin para expresar esa problemática, considerando estas palabras del escritor británico como poseedoras de un marchamo de verdad: "Aquella virtud de la originalidad que los hombres tanto enfatizan no es la novedad (como ellos vanamente piensan), sino que es simplemente autenticidad [genuineness]; depende por completo de esta sola y gloriosa facultad que consiste en alcanzar los resortes de las cosas y lograr comprender desde ellos» ${ }^{10}$. En estos mismos años de estancia bengalí, Coomaraswamy aludió a la cuestión de la originalidad como si estuviera leyendo en clave india -y en medio de su época más combativamente anticolonial- los planteamientos de Ruskin en torno a las facultades de la imaginación recogidas en el segundo volumen de Modern Painters, como en este fragmento del ensayo «Art and Yoga in India» (1909):

Perseguir la originalidad, como el mismo Novalis bien percibió, es egoísmo. Mucho mejor sería echarse a un lado de este 'burdo egoísmo', y mucho más satisfactorio si, mediante la fuerza de la imaginación podemos captar y representar alguna sombra de las grandiosas realidades que los artistas de todas las épocas han visto y escuchado. Y esta intensidad imaginativa es la verdadera inspiración [Coomaraswamy juega aquí con las palabras inspiration e in-breathing]; que implica apartar el yo más insignificante para dejar paso a un yo más exuberante, en contacto con una realidad más real. Y esto, una vez más, es yoga (Coomaraswamy, 1981: 60).

John Ruskin expresaba la esencia de la originalidad no como novedad sino como autenticidad. Y, en franca proximidad a él, Coomaraswamy hablaba de la imaginación como el recurso que abre la originalidad a un campo de inspiración verdadera, asociándolo al yoga ${ }^{11}$. A través de esta forma de presentar la inspiración y los modos «indianizados» de originalidad, Coomaraswamy remite a lo tradicional en el sentido de autenticidad anteriormente apuntado, pero sobrepuesto a una cualidad específica de sacralidad. Los atributos transcendentalistas y espirituales pasaron a formar parte de un forzado discurso de oposición a la deriva artística contemporánea de Occidente; el atributo metafísico sobrepuesto al arte asiático se oponía al materialismo propio del arte decimonónico europeo, y en especial a la apología del naturalismo como esencia del arte occidental. En este sentido, la paradoja anteriormente apuntada al hilo de la utilización de ciertos segmentos del pensamiento de John Ruskin por parte de Coomaraswamy es especialmente llamativa, y lo es por constituir precisamente Modern Painters de Ruskin uno de los mayores monumentos teóricos a ese naturalismo respecto del cual el historiador cingalés buscaba una antítesis viable en el contexto indio.

En esta construcción de un movimiento artístico renovador bengalí en hermanamiento con los valores panasiáticos japoneses, la búsqueda de una burbuja teórica y estética que pudiera englobar a ese segundo vértice del triángulo de Okakura -la «tradición»-, es indisociable del vuelco condicionado hacia los otros dos: por un lado, hacia la búsqueda de acomodo de una forma de relación creativa con la naturaleza, y por otro, hacia la participación de un ideal de originalidad también marcado (a pesar de los intentos por separar ambas actitudes) por la búsqueda de la novedad. Partha Mitter ha situado precisamente en las sinergias de Okakura Kakuzō con el svadeśi artístico ben- 
galí el origen de las claves de progreso e individualismo que la Escuela de Bengala empezó a poner silenciosamente en práctica (Mitter, 1994: 265). Ese tipo de actitudes, fruto necesario del impacto con el pensamiento artístico occidental-moderno, encontraron acogida con mayor facilidad en la Bengala svadeśí cuando era un japonés el que las presentaba según una tríada como naturaleza-tradición-originalidad, entendida como un ideal estético esencialmente consonante con el sentir artístico asiático y como una respuesta selectiva a la occidentalización (Mitter, 2007: 80). A tenor de lo expuesto, el ideal triangular de Okakura se está contemplando aquí no simplemente a raíz de que el crítico japonés dejara constancia explícita de él en ninguno de sus textos -hecho que aparentemente no ocurrió-, sino porque hizo fortuna de manera formidable en la literatura artística de Nandalal Bose y de Kalābhavan, y posteriormente en la historiografía específica. Resulta especialmente interesante que un comentario, una apreciación formativa que Okakura Kakuzō compartió en privado con el estudiante Nandalal Bose en 1912, adquiriera el peso que posteriormente tuvo. La razón quizás resida en la versatilidad y maleabilidad de las relaciones asumibles entre esos tres elementos, pues, a la luz de las claves analizadas a lo largo de este capítulo, el ideal naturaleza-tradición-originalidad empieza a mostrar un carácter menos compacto, más contradictorio y fragmentario de lo que cabría esperar; pues lo previsible aquí no es otra cosa que la imposible naturaleza maciza de lo auténtico (su ficticia univocidad), y lo obtenido es la versatilidad necesariamente discordante que se oculta bajo sus mantos.

Las pistas que aporta ese triángulo, y especialmente su exégesis, son tanto transparentes hacia las inquietudes de los teóricos y artistas bengalíes como esclarecedoras acerca de los mimbres con los que fueron construidos los principales textos teóricos combativos del arte asiático: los de Okakura Kakuzō y de Ananda Coomaraswamy.

Como ya se ha apuntado, esa interacción nació en Calcuta de la mano del eslabón de la familia Tagore, pero su proyección no era exclusivamente bengalí sino que se dirigía en última instancia hacia el Atlántico norte y acabó encontrando su acomodo en otra capital: Boston. La inspiradora red de contactos de Calcuta permitió fraguar en los escritos de Okakura y Coomaraswamy una mirada renovada hacia el arte asiático, a la vez combativa hacia las distorsiones y excesos de la extensión cultural colonial, y sugestiva de cara al interlocutor occidental. Solo desde ese doble funcionamiento puede entenderse que ambas figuras, fuertemente beligerantes en sus escritos con la modernidad occidental, pudieran acabar formando parte del Departamento de Arte Oriental del Museo de Bellas Artes de Boston en dos fases diferentes (1905-13 en el caso de Okakura Kakuzō, desde 1917 en el caso de Coomaraswamy). Esa aparente coincidencia habla en realidad de un relevo relativo a la unidad asiática pregonada por Okakura en aquellos años 190203. La posición teórica de Coomaraswamy con respecto al arte indio, pero también más ampliamente, asiático, es fundamental para comprender el destino ideológico final que esa unidad que pregonada en The ldeals of the East acabó alcanzando. Las primeras frases de The Transformation of Nature in Art de Ananda Coomaraswamy, publicadas en Harvard en 1934, cuentan con una contundencia casi equiparable a la del manifiesto de Okakura, aunque en este caso apelan a una unidad no exclusivamente asiática, sino culturalmente global. En aquellos momentos Ananda Coomaraswamy había abrazado ya el concepto de la Philosophia Perennis, que aportaba a las tendencias tradicionalistas de todas las partes del mundo una capacidad de trascendencia totalmente convergente. La Edad Media había sido, según Coomaraswamy el último momento de verdadera conexión entre los extremos geográficos: «hubo un tiempo en que Europa y Asia podían y de hecho se entendían perfectamente. Asia ha permanecido la misma; pero subsecuentemente a la extroversión de la consciencia europea y a su preocupación por las superficies, ha llegado a ser cada vez más difícil para las mentes europeas pensar en los términos de unidad" (Coomaraswamy, 1997: 7). La unidad referida por el autor cingalés, en plena consonancia con la Philosophia Perennis, tenía un calado eminentemente espiritual, como inicialmente lo tuvo también el discurso de Okakura. Ambos eran obviamente conscientes de las ansias de renovación espiritual de los sectores occidentales más comprometidos con la modernidad desde finales del siglo XIX, volcando sus anhelos en las enseñanzas venidas de Oriente y canalizadas principalmente a través de la Teosofía. Coomaraswamy rechazaba en ese mismo pasaje que esa unidad mundial existiera en su tiempo a consecuencia de los cambios de la Europa post-renacentista, frente a la «estabilidad» histórica de Asia. Sin embargo, lo que Ananda Coomaraswamy no reconoce es que esa misma «extroversión» europea fue la que permi- 
tió la articulación ideológica de la unidad misma de Asia, y la estabilidad de Oriente no era tal, habida cuenta de la disrupción sin parangón de la cultura occidental en las sociedades india y japonesa de la que eran plenos deudores los protagonistas que han sido referidos a lo largo de este texto. En otras palabras, el tradicionalismo esgrimido por Okakura Kakuzō, el núcleo bengalí en torno a la familia Tagore, y el propio Ananda Coomaraswamy en último término, no remitía tanto a las formas artísticas de la tradición japonesa o india como a la imagen seductora del pasado asiático dirigida al público occidental. Dicha imagen era asimilable, y su unidad creíble, precisamente por haber sido creada por herederos de la cultura colonial, con instrumentos ideológicos de la modernidad occidental, desde un discurso dialéctico al mismo tiempo beligerante y seductor. La variedad de los ideales asiáticos incorporaba ya componentes externos, y eran todos ellos los que permitían tejer a medida un nuevo ideal de unidad.

\section{Notas}

1 Se ha preferido utilizar en este texto la forma japonesa, apellido + nombre, para nombrar a Okakura Kakuzō, dado que se encuentra referido de esta manera en la mayoría de las fuentes consultadas.

2 Okakura Kakuzō (1862-1913) fue una de las personalidades más importantes de la cultura japonesa en el cambio de siglo. Recibió una formación occidental a pesar de proceder de una familia fuertemente tradicional, fruto de la rápida y radical modernización acaecida durante el periodo Meiji (1868-1912). Viajó por Europa y Estados Unidos como emisario cultural del gobierno japonés antes de postularse como principal ideólogo y promotor de la corriente más tradicionalista de las nuevas artes japonesas, el Nihonga. Es especialmente famoso por haber escrito El libro del té (The book of tea, Londres, 1906), considerado un auténtico manifiesto de la identidad de la estética japonesa y de la superioridad moral de su país frente a Occidente. Por otra parte, Ananda Coomaraswamy (1877-1947) fue un historiador del arte nacido en Colombo (Ceilán, hoy Sri Lanka) de padre cingalés de origen indio y de madre británica. A pesar de haber recibido inicialmente una formación científica, pronto orientó su actividad hacia la crítica cultural nacionalista en India, y posteriormente hacia el estudio del arte y la filosofía del Subcontinente indio.

3 La proclama anticolonial de Okakura fue pervertida por el nacionalismo japonés de los años 30 y utilizada en clave imperialista, convirtiendo por tanto el espíritu de resistencia del pueblo asiático en un argumento del paternalismo nipón sobre el resto del continente. Véase: Notehelfer, $1990: 331$.

4 Satyajit Ray teorizó acerca de la unidad estética del cine asiático en textos como "Calm without, Fire within», encontrando afinidades con Japón en el carácter y «temperamento» del artista-cineasta oriental, representado metafóricamente por el Monte Fuji. Ray, 1993: $152-157$.

5 Los términos literales y en el orden expresado por Abanindranath Tagore serían tradition (țryāọiśan, ট্র্যাডশিন), observation (abasārbheśan, অবসার্ভশেন) y originality (orijināliți, ওরজিনিলটি). Tagore, A., 1944: 106.

6 El concepto de rasa es fundamental en la tradición estética india, siendo objeto continuo de comentarios por parte de los pensadores indios de la Edad Media, y siendo especialmente estudiado desde principios del siglo XX. El concepto de rasa ha sido objeto de interpretaciones fuertemente ideologizadas en el contexto colonial, como es el caso de Ananda Coomaraswamy. Para un acercamiento al concepto de rasa, ver: Maillard y Pujol (1999).

7 Ananda Coomaraswamy a menudo diferenciaba el tipo de artista «normal» (asiático, medieval europeo) del «anómalo» (post-renacentista, moderno, sentimental): «Nuestra forma de entender el arte, como, esencialmente, la expresión de la personalidad, nuestra concepción del genio, nuestra impertinente curiosidad por la vida privada del artista, todas estas cosas son producto de un individualismo pervertido y nos impiden comprender la naturaleza del arte medieval y oriental. La manía moderna de la atribución es la expresión del engreimiento del Renacimiento y del humanismo del siglo XIX; no tiene nada que ver con la naturaleza del arte medieval, y se convierte en un error sentimental cuando se le aplica". Coomaraswamy, $1980: 44$.

8 En «About Pictures», un texto elaborado en su estancia bengalí de 1910 y publicado en la Modern Review de Calcuta, contraponía originalidad y novedad: «Un arte puramente imitativo, aunque pueda resultar bello, con razón podría ser considerado arcaico. Pero incluso esto es mejor que confundir originalidad con novedad» (Coomaraswamy, 1910: 594). Años más tarde, en un texto sobre Nietzsche, Ananda Coomaraswamy volvía sobre la misma idea: según este autor, tanto Nietzsche como William Blake y Walt Whitman representarían una interesante posición individualista occidental (Individualismo Idealista lo llama Coomaraswamy), marcada por una «originalidad que no consiste en una incomprensible y antinatural novedad»; en «Cosmopolitan view of Nietzsche», recogido en Coomaraswamy, 1918: 115.

9 La oposición de originalidad y novedad era un tema de debate bastante habitual, al que también se refirió en más de una ocasión Rabindranath Tagore. En una conferencia de 1929 Tagore aludió a esa cuestión, y diferenciando y oponiendo ambos conceptos (Tagore, R, 1929: 6). En una conversación epistolar con William Rothenstein (carta enviada desde Śāntiniketan el 25 de julio de 1919) Tagore comentaba que la literatura moderna solo persigue la originalidad entendida como novedad, que no puede ser verdadera originalidad. En tal apreciación parece seguir (casi usando las mismas palabras) a Ruskin en Modern Painters, en la misma referencia que se cita a continuación en el cuerpo del texto. La carta de Tagore se encuentra recogida en: Lago, $1972: 257-258$.

10 La cita utilizada por Coomaraswamy procede del segundo volumen de Pintores Modernos: Ruskin, 1903: 178. Coomaraswamy hacía uso de esta cita en un texto sobre «Los objetivos y métodos del arte indio" (1909), arte del cual Ruskin era un reconocido detractor, por lo cual no deja de ser paradójico que Coomaraswamy recurra a él en un texto reivindicativo de la especificidad del arte indio (Coomaraswamy, 1981: 42).

11 La explicación del arte como yoga es una constante en Coomaraswamy y nace del afán por presentar la práctica del artista indio tradicional en términos unitarios, como una superación de las diferencias entre sujeto y objeto (Coomaraswamy, 1996: 20; Fernández del Campo, 2013: 28-36). No obstante, en fases posteriores del pensamiento de Coomaraswamy, que acabaron por convertirse en canónicas dentro de su pensamiento y hegemónicas con respecto a la trayectoria del arte indio, el autor cingalés no terminó por concebir la originalidad en términos positivos. A su modo de ver era la causa de la pérdida de función y sentido de las creaciones artísticas: KAPUR, Geeta, "On the Vocation of the Artist», en Sheikh, Subramanyan y Vatsyayan, 1984 : 151. 


\section{Bibliografía}

BHARUCHA, Rustom (2010), Another Asia. Rabindranath Tagore \& Okakura Tenshin, Oxford University Press, Nueva Delhi.

CHAKRABARTY, Dipesh (2008), Al margen de Europa. Pensamiento Postcolonial y diferencia histórica (tr. de Alberto Álvarez y Araceli Maira), Tusquets, Barcelona.

CHATTERJEE, Partha (2011), Nationalist thought and the Colonial World. A derivative discourse, University of Minnesota Press, Minneapolis. CLARK, John (2005), «Okakura Tenshin and Aesthetic Nationalism», East Asian History, n. ${ }^{\circ}$ 9, pp. 1-38.

COOMARASWAMY, Ananda K. (1910), «About pictures», The Modern Review, vol. VIII, n. 5 (pp. 523-524) y n. ${ }^{\circ} 6$ (pp. 592-594).

COOMARASWAMY, Ananda K. (1918), The Dance of Śiva. Fourteen Indian Essays, The Sunwise Turn, Nueva York.

COOMARASWAMY, Ananda K. (1980), La filosofía cristiana y oriental del arte (tr. de Esteve Serra), Taurus, Madrid.

COOMARASWAMY, Ananda K. (1981), Essays in National Idealism, Munishram Manoharlal, Nueva Delhi.

COOMARASWAMY, Ananda K. (1996), La danza de Śiva. Ensayos sobre arte y cultura india (ed. Eva Fernández del Campo), Siruela, Madrid. COOMARASWAMY, Ananda K. (1997), La transformación de la naturaleza en arte, Kairós, Barcelona.

DEPARTMENT OF HISTORY OF ART (KALABHAVANA) (1979), "Kala-Bhavana - Then and Now. A Special Issue», Nandan. An Annual on Art and Aesthetics.

FERNÁNDEZ DEL CAMPO, Eva (2013), Los cánones del arte indio, Abada, Madrid.

FERNÁNDEZ GÓMEZ, Rosa (2008), «La estética invisible del arte popular», Contrastes, Revista Internacional de Filosofía, suplemento 13, pp. 41-54.

GUHA-THAKURTA, Tapati (2008), The Making of a New 'Indian' Art. Artists, Aesthetics and Nationalism in Bengal, c. 1850-1920, Cambridge University Press, Cambridge.

GUHA-THAKURTA, Tapati (2009), «Dialogues in Artistic Nationalism», Art India. The Art News Magazine of India, vol. XIV, n. ${ }^{\circ}$ 3, pp. $22-43$.

GUTH, Christine M. E. (2000), "Charles Longfellow and Okakura Kakuzō: Cultural Cross-Dressing in the Colonial Context», Positions, vol. $8, n .^{\circ} 3$, pp. $605-636$.

HAY, Stephen N. (1970), Asian Ideas of East and West. Tagore and his critics in Japan, China and India, Harvard University Press, Cambridge (Mass.)

LAGO, Mary M. (ed.) (1972), Imperfect Encounter. Letters of William Rothenstein and Rabindranath Tagore, 1911-1941, Harvard University Press, Cambridge (Mass.).

MAILLARD, Chantal y PUJOL, Óscar (1999), Rasa. El placer estético en la tradición india, Indica Books, Varanasi.

MITTER, Partha (1994), Art and Nationalism in Colonial India, 1850-1922. Occidental orientations, Cambridge University Press, Cambridge. MITTER, Partha (2007), The Triumph of Modernism. India's artists and the avant-garde, 1922-1947, Reaktion Books, Londres.

NAGAHIRO, Kinoshita (2012), "Okakura Kakuzō as a Historian of Art», Review of Japanese Culture and Society, vol. 24, n. ${ }^{\circ}$, pp. 26-38 (DOI: 10.1353/roj.2012.0010).

NINOMIYA-IGARASHI, Masumi (2010), Drawn toward India: Okakura Kakuzō's Interpretation of Rájendralála Mitra's Work in his construction of Pan-Asianism and the History of Japanese Art (tesis doctoral), University of North Carolina.

NOTEHELFER, F. G. (1990), «On Idealism and Realism in the Thought of Okakura Tenshin», Journal of Japanese Studies, vol. 16, n. ${ }^{\circ}$, pp. 309-355.

OKAKURA, Kakuzo (1905), The Ideals of the East, with special reference to the art of Japan, John Murray, Londres.

OKAKURA, Kakuzo (2018), Los ideales de Oriente: con especial referencia al arte japonés (tr. de Fernando Álvarez), Satori, Gijón. RAY, Satyajit (1993), Our Films, Their Films, Longman, Calcuta.

ROMÁN, Sergio (2013), «La enseñanza del arte en Santiniketan como punto de encuentro de Japón y Europa bajo el amparo ideológico de Rabindranath Tagore», en GARCÉS GARCÍA, Pilar y TERRÓN BARBOSA, Lourdes (eds.), Itinerarios, viajes y contactos Japón-Europa, Peter Lang, Berna, pp. 787-795.

RUSKIN, John (1903), Modern Painters, vol. Il (Of the Imaginative and Theoretic Faculties), George Allen, Londres.

SAMANTA, Sri Kanai (1982), «Nandalal Bose on Art», Visva-Bharati News (Nandalal Bose Centenary Number 1882-1982), pp. 4-17. 
SHEIKH, Gulam Mohammed, SUBRAMANYAN, K. G., VATSYAYAN, Kapila (1984), Paroksa. Coomaraswamy Centenary Seminar Papers, Lalit Kala Akademi, Nueva Delhi.

TAGORE, Abanindranath (1944), Jorāsāñkkor Dhāre, Kalikātā, Biśvabhāratī Granthālaỳa.

TAGORE, Rabindranath (1929), «The Philosophy of Leisure», The Visva-Bharati Quarterly, vol. VII, n. ${ }^{\circ}$ 1, pp. 5-12.

VATSYAYAN, Kapila (1984), "Nature, Tradition and Originality: A Paradigm for Art Historical Study», The Visva-Bharati Quarterly, vol. 49, n. ${ }^{\circ}$ 1-4, pp. 142-163. 